\title{
Endoscopic Manifestation in HIV Patients Presenting with Gastrointestinal Symptoms: A Descriptive Study.
}

\author{
John Paul ${ }^{*}$, Sara Chandy** \\ *Resident. Department Of General Medicine, St. Johns Medical College Hospital \\ ${ }^{* *}$ Associate Professor Department Of General Medicine, St. Johns Medical College Hospital,
}

\begin{abstract}
Human immunodeficiency virus (HIV) infection and acquired immune deficiency syndrome (AIDS) due to its varied presentations and multisystem involvement has become a major cause of mortality and morbidity encountered in practice of modern medicine. A large proportion of patients with HIV-AIDS present with a wide spectrum of gastrointestinal symptoms during the course of the disease ${ }^{l}$.Studies on the prevalence of GI symptom, diagnosis, endoscopic findings and management in the Indian population is lacking. Hence we studied the clinical and endoscopic manifestations in HIV infected patients with upper GI symptoms.

This is a cross sectional descriptive study of upper endoscopic findings in HIV infected patients presenting with upper gastro-intestinal symptoms to the tertiary center multi-specialty hospital of 1100 beds. We studied 55successive HIV infected patients who underwent Esophagogastroduodenoscopy from 1 september 2011 to 31 august 2012.

The mean age of the subjects was 43.76 years. There were 37 (67.27\%) males and $18(32.73 \%)$ females. In our study, of the 55 patients $30(70.91 \%)$ had a CD4 count of $<200$. While 11(20\%) had a CD4 count between 200-499, 5(9.09\%) patients had a CD4 count of 500 or less. Dysphagia(26\%) was found to be the most commonly appearing symptom followed by vomiting(23\%), dyspepsia(19\%),nausea(19\%) and epigastric pain(12\%).Idiopathic oesophageal ulceration was the most common pathology noted followed by CMV, Hiatus hernia, Oesophageal candidiasis and Oesophageal varices .Idiopathic oesophageal ulceration though not being among the opportunistic infections was the most common finding in our study. There was no significant correlation between the presence of an endoscopic finding with CD4 counts. However, in our study, we noted that there was a significant correlation between clinical stage and probability of a positive endoscopic finding. Idiopathic Oesophageal Ulceration was found to be the most common endoscopic finding among patients with HIV presenting with upper GI symptom. This study has noted that oesophageal candidiasis was not the most common endoscopic finding among HIV patients with upper GI symptoms. This could be attributed to empiric treatment with antifungals and early diagnosis and optimal treatment of $H I V$. Hence even though the prevalence of oesophageal candidiasis might remain the same, it is less often seen during regular endoscopies than before.CMV involvement of the upper GI tract was found to be more common in patients on HAART unlike the rest of the endoscopic findings. This could be attributed to the relatively longer mean duration of the disease and the significantly lower CD4counts in this subset. Treatment failure and progression of the disease could have contributed to this situation. Endoscopic findings increase as the disease progresses.
\end{abstract}

\section{Introduction}

The worldwide scourge of human immunodeficiency virus (HIV) infection and acquired immune deficiency syndrome (AIDS) has impacted profoundly the practice of medicine. According to National AIDS Control Organization (NACO) estimates there are about 2.3 million people infected in the country. The GI tract has long been recognised as a major site of HIV related diseases and up to $90 \%$ of HIV infected patients experience GI symptoms during the course of their disease ${ }^{1}$.However, studies on the prevalence of GI symptom, diagnosis, endoscopic findings and management in the Indian population is lacking. HIV infection leads to progressive deterioration of both the general and the local immune defence mechanisms of the GI tract mucosa ${ }^{2}$. Opportunistic infections remain a major cause of morbidity and account for the high prevalence of dysphagia, odynophagia, abdominal pain, hematemesis and other common upper gastrointestinal symptoms in HIV infected patients. EGD is very useful tool for the early diagnosis of opportunistic infections, nonspecific inflammatory conditions, and other GI associated symptoms encountered in this population. Hence we studied the clinical and endoscopic findings in HIV infected patients with upper GI symptoms.

\section{Materials And Methods}

This was a cross sectional descriptive study. HIV positive adults confirmed by ELISA presenting with significant upper GI symptoms were included in the study following a valid consent. Data was collected 
based on the proforma prepared for the study. HIV patients referred from inpatient and outpatient departments with upper GI symptoms for endoscopic evaluation were considered for the study. Patient was interviewed and examined based on a predesigned proforma.The Edmonton Symptom Assessment System (ESAS) was used for assessing the severity of upper gastrointestinal symptoms. It is a selfreporting tool of symptom intensity and is designed to enable repeated quantitative measurements of symptom intensity with minimal patient burden. Each symptom (i.e. nausea, vomiting, epigastric pain. etc) is rated along a numerical scale, ranging from 0 (not at all, no symptom or best) to 10 (worst possible) ( $^{3}$.In our study, the symptom was considered significant if the severity score was $\geq 4$. These patients were endoscoped (instrument used OLYMPUS V 140/160/180 ) and material for cytological examination were obtained using standard endoscopic brush, slides prepared and screened for fungal elements and both Herpetic and CMV inclusions. Biopsies of oesophageal mucosa were taken from the edge of any ulcer present. The biopsies were fixed in $15 \%$ formal saline, embedded in paraffin. 4 micrometre sections were cut from at least three levels and routinely stained with haematoxylin and eosin, Periodic acid Schiff for fungi and ZiehlNeelsen stains for mycobacteria ${ }^{4}$. The endoscopic and histopathological diagnoses were recorded. Statistical analysis was done using SPSS 16. Results on continuous measurements are presented as Mean+/- SD. Students $\mathrm{T}$ test used to measure significance of differences between means of numerical data. Chi square test was used to assess correlations between categorical variables.

\section{Observation}

The mean age of the subjects was 43.76 years. There were $37(67.27 \%)$ males and $18(32.73 \%)$ females. The male to female ratio (2.05) was in fact higher than the national male to female ratio (1.6). This could be due to the small sample size or may indicate that a lower proportion of female patients report to the hospital or have access to hospital when they have GI symptoms in the background of HIV infection. Dysphagia(26\%) was found to be the most commonly appearing symptom followed by vomiting $(23 \%)$, dyspepsia(19\%),nausea(19\%) and epigastric pain(12\%).

Figure 1: Endoscopic findings

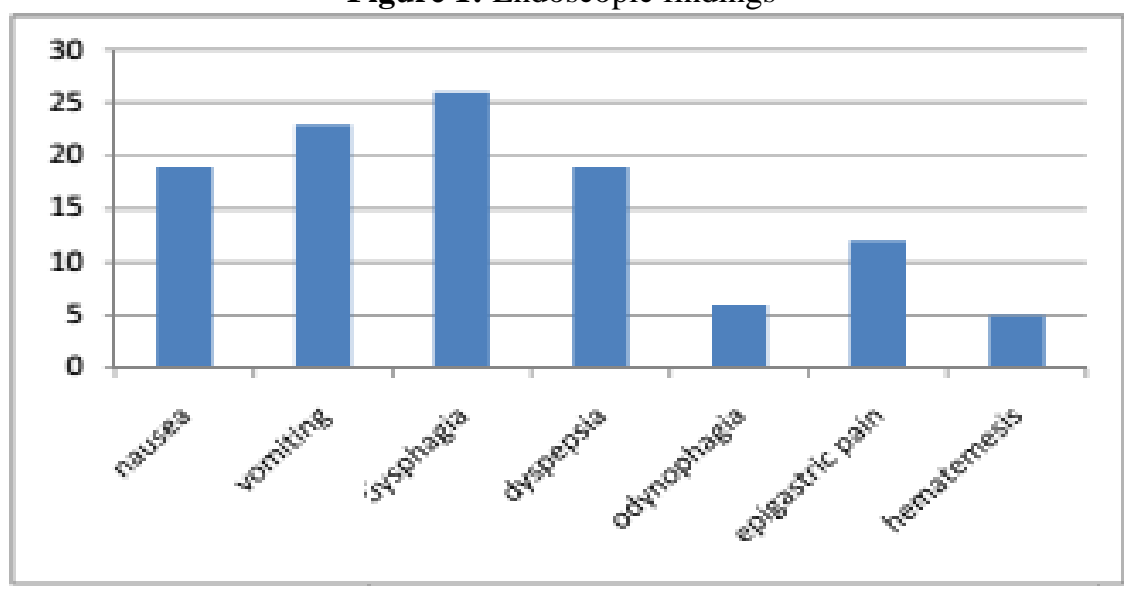

Table 1 : Endoscopic findings

\begin{tabular}{|l|l|}
\hline ENDO & Number (Percentage) \\
\hline Normal & $22(40.00)$ \\
Esophageal candiditasis & $3(5.45)$ \\
Idiopathic ulcers & $12(21.82)$ \\
Varices & $2(3.64)$ \\
CMV & $5(9.09)$ \\
Hiatus hernia & $4(7.27)$ \\
Others & $7(12.73)$ \\
\hline
\end{tabular}


Idiopathic oesophageal ulceration was the most common pathology noted followed by CMV,Hiatus hernia ,Oesophageal candidiasis and Oesophageal varices .Idiopathic oesophageal ulceration though not being among the opportunistic infections was the most common finding in our study. Numerous trials have demonstrated a reduction in the incidence of most opportunistic infections since HAART was introduced. ${ }^{5,6,8}$ Empiric treatment of HIV patients presenting with upper gastro-intestinal symptoms with oral fluconazole could have led to the decreased frequency of oesophageal candidiasis as an endoscopic finding, however the disease prevalence might have been the same.

On comparing the various endoscopic findings, there was no significant difference in the presence of an endoscopic finding whether the patient was on HAART or not. Reason for this observation could be the small sample size with most of them being newly diagnosed cases of HIV yet to be started or recently started on Haart.

In our study we observed that oesophageal diseases due to infective causes(candidiasis, CMV etc.) were noted predominantly in patients with CD4 counts less than $200 / \mu 1$ where as non infective causes(hiatus hernia,varices..etc) predominated in patients with CD4 counts more than 200/ $\mu 1$.While there was no significant correlation between the presence of an endoscopic finding in HIV with CD4 counts, a study done over a longer period with a much larger number of subjects would be needed to show significant differences in endoscopic manifestations in different CD4 subgroups.

Table 2 : Comparison of endoscopic findings between patients on ART and ART

naïve.

\begin{tabular}{|l|l|l|l|}
\hline ART category & \multicolumn{2}{|l|}{ ENDO findings category } & \\
& \multicolumn{2}{|l|}{ p-value } \\
\cline { 2 - 4 } & Absent & Present & \\
\hline Absent & $9(40.91)$ & $16(48.48)$ & 0.580 \\
Present & $13(59.09)$ & $17(51.52)$ & \\
\hline
\end{tabular}

Table 3:Association between endoscopic findings between different clinical stages of HIV.

\begin{tabular}{|c|c|c|c|}
\hline \multirow[t]{2}{*}{ Clinical Stage } & \multicolumn{2}{|c|}{ ENDO category } & \multirow{2}{*}{ p-value } \\
\hline & Absent & Present & \\
\hline Stage1 & $7(31.82)$ & $8(24.24)$ & \\
\hline Stage2 & $15(68.18)$ & $15(45.45)$ & 0.029 \\
\hline Stage3 & $0(0.00)$ & $2(6.06)$ & \\
\hline Stage4 & $0(0.00)$ & $8(24.24)$ & \\
\hline
\end{tabular}

However, in our study, we noted that there was a significant correlation between clinical stage and probability of a positive endoscopic finding.

\section{Conclusions}

Idiopathic Oesophageal Ulceration was found to be the most common endoscopic finding among patients with HIV presenting with upper GI symptom This study has noted that oesophageal candidiasis was 
not the most common endoscopic finding among HIV patients with upper GI symptoms. This could be attributed to empiric treatment with antifungal along with early diagnosis and optimal treatment of HIV.Hence even though the prevalence of oesophageal candidiasis might remain the same, it is less often seen during regular endoscopies than before. Endoscopic findings increases as the disease worsen as increasing immunosuppression leads to changes in the gastric mucosal immunity and thereby predispose to opportunistic infections.

\section{References}

[1]. Lorenz KA, Shapir MF,Asch SM, Bozzete SA, Hays RD. Association of symptoms and health related quality of life: Findings from a National study ofpersons with HIV infection. Ann Intern Med. 2001; 134:854-68.

[2]. Weber R, Ledergerber B, Zbinden R, Altwegg M, Pfyffer GE, Spycher MA et al. Enteric infections and diarrhoea in human immunodeficiency virus-infected persons: prospective community-based cohort study. Swiss HIV Cohort Study. Arch Intern

[3]. Med 1999;159:1473-1480.

[4]. Bruera E, Kuehn N, Miller MJ, Selmser P, Macmillan K. The Edmonton Symptom Assessment System (ESAS): a simple method for the assessment of palliative care patients. J Palliat Care 1991;7:6-9.

[5]. Wilcox CM, Saag MS. Gastrointestinal complications of HIV infection: changing priorities in the HAART era. Gut 2008;57:861870 .

[6]. Bashir, Roshan M, Wilcox C, Rabeneck, Linda. Symptom-Specific Use ofUpper Gastrointestinal Endoscopy in Human Immunodeficiency Virus-Infected Patients Yields High Dividends. J of Clin Gastroentero 1996;23(4):292-298.

[7]. Monkemuller KE, Call SA, Lazenby AJ, Wilcox CM. Declining prevalence of opportunistic gastrointestinal disease in the era of combination antiretroviral therapy. Am J Gastroenterol 2000; 95: 457-462.

[8]. Palella FJ, Delaney KM, Moorman AC. Declining morbidity and mortality among patients with advanced human immunodeficiency virus infection. N Engl J Med 1998; 338: 853-860.

[9]. Mocroft A, Oancea C, van Lunzen J, Vanhems P, Banhegyi D, Chiesi A et al. Decline in esophageal candidiasis and use of antimycotics in European patients with HIV. Am J Gastroenterol 2005; 100: 1446-1454. 